\title{
Intelligent Condition Monitoring Techniques for Early Fault Diagnosis of Rotating Machines - A Review
}

\author{
Vinod Kumar ${ }^{1}$, Deepam Goyal ${ }^{2}$, S.S Dhami ${ }^{1}$, \\ ${ }^{1}$ Department of Mechanical Engineering, National Institute of Technical Teachers Training \& Research, \\ Chandigarh,160019, India \\ ${ }^{2}$ Department of Mechanical Engineering, Chitkara University, Punjab,140401, India
}

\begin{abstract}
Condition-based maintenance is always an important strategy of maintenance to prolong the effective life of rotating machines as they run on high speeds with a variety of loads in some cases under severe conditions. If the monitoring of the current condition is not done accurately then rotating machines such as turbines, engine, bearing, shafts, gearbox, motors, and compressors leads to catastrophic failures with some serious consequences on the rate of production, safety, loss of manpower and sudden increase in repairing cost. Condition-based maintenance is also be called predictive type maintenance is a far superior technique as compared to preventive maintenance and run-to-break maintenance. In predictive maintenance current status of the machine while in operation, being monitored carefully and based on a brief analysis of the future condition of the machine predicted. This paper going to review the various intelligent condition monitoring techniques developed or used by various researchers to monitor the health of rotating machines and able to predict the faults at the earliest time.
\end{abstract}

Keywords Rotating Machines, Fault Diagnosis, Vibration Analysis, Condition Monitoring,

\section{INTRODUCTION}

Almost all rotating equipment's are subjected to various kinds of conditions such as loading, unloading, change in speed, change in other working parameters, and change in working environments also. All this greatly affect the machine performance and so to improve this it is always required to monitor the current status of the machine and implement certain maintenance techniques according to that. In this modern time, we are living with a revolution going in various service sectors as industry 4.0 currently changes the scenario of maintenance techniques used in various industries. Now the condition of various machines can be checked remotely with the help of various soft computing techniques, new artificial intelligence techniques developed and used to diagnose the problem or even now prognostic approaches are now very famous in large industries like manufacturing, power and energy, oil, and gas industries [1]. There are various kinds of condition monitoring techniques that are used by various organizations, some of them are listed as vibration analysis, lubricant analysis, performance analysis, thermography, noise inspection etc [2]. But condition monitoring using vibration as a tool for diagnostic is the most prevalent technique for machine health monitoring [3]. As it is immediately reacted to change so condition monitoring by using vibration technique can be used for intermittent monitoring or permanent monitoring. While if we go through oil analysis several days may be spent with their collection and their analysis although some online techniques do exist. One major problem that comes with oil analysis is of components particularly with bearings having a similar chemical composition, then it became difficult to find the faulty component [4]. But there is 
no issue with vibration analysis as it helps to find the faulty component in a machine by using amplitude or frequency features having distinct nature. So, it is easy to diagnose the fault with the help of vibration signatures. Now with the passage of time various powerful signals processing techniques developed which enables the extraction of very weak signal of fault from masking or noise signals [5]. In condition monitoring of machine change in the signature of vibration, signals give rise the indication about the health of the machine. So, it becomes equally important to find the factors that cause the change in vibration signals and then either reduced or eliminate them [6]. There is a number of factors that affect the change in vibration signal of rotating machines such as speed, load, unbalance, misalignment, bent shaft, cracked shafts etc. All these mentions factors in rotating machines provide distinct vibration signatures furthermore they can be analysed by various signal processing techniques, and thus help to identify the health of machine tools. In the past condition, monitoring is used to detect the faults in machines and to know critical operation conditions, but now in modern times conditions monitoring is also be done to utilizing the available resources in the most efficient ways [7]. As rotating machines run at high speed with various load conditions, they can easily be subject to various kinds of faults with different levels of severities that take place in the rotating machine and if they were ignore caused catastrophic failure and increase the downtime all such faults are listed below in table 1.

Table 1: Some typical defects and faults detected with vibration analysis.

\begin{tabular}{|l|l|}
\hline Item & Fault \\
\hline Gears & $\begin{array}{l}\text { Tooth messing faults, misalignment, cracked and/or worm teeth, eccentric } \\
\text { gear }\end{array}$ \\
\hline Rotors and shaft & $\begin{array}{l}\text { Unbalance, bent shaft, misalignment, eccentric journals, loose components, } \\
\text { rubs, critical speed, cracked shaft, blade loss, blade resonance }\end{array}$ \\
\hline Rolling element bearings & Pitting of race and ball/roller, spalling, other rolling elements defect \\
\hline Flexible coupling & Misalignment, unbalance \\
\hline Electrical machines & $\begin{array}{l}\text { Unbalanced magnetic pulls, broken/damaged rotor bars, air gap geometry } \\
\text { variations, structural and foundation faults, structural resonance, piping } \\
\text { resonance, vortex shedding }\end{array}$ \\
\hline
\end{tabular}

\section{DETECTION OF FAULT AND DIAGNOSTIC WITH VIBRATION ANALYSIS}

Diagnosis is specified as determining the condition of a machine based on symptoms. Diagnosis needs the ability to recognise the status of the machine based on symptoms. In this context, the term diagnosis is used in the same way as it is used in medicine. Vibration is commonly seen as an indicator of a gearbox problem. The vibration produced by gearboxes is complex in structure, yet it contains a lot of information. We can consider vibration to be an indicator of a gearbox malfunction. To identify and diagnose an imminent failure, a detailed study of the evidence relevant to the catastrophic failure, as well as procedures for gathering and quantifying the information, is required. Even though many faults could be readily identifiable by physical assessment of a component employing techniques such as X-ray microscopy, the dye penetrates and many more, all such methods are usually not to be used because they can cause physical damage to machine components or require some structural change in machine assembly [8]. Gears are commonly used in machines to transfer power from one neighbouring shaft to another with changes in torque and speed. Most gears having a conjugate profile such that there will be a constant output speed if there is a constant input speed [9]. There are several methods to diagnose the faults in rotating machines and these are 
shown in figure 2.1, some of them are signal based, model-based, knowledge-based and hybrid methods.

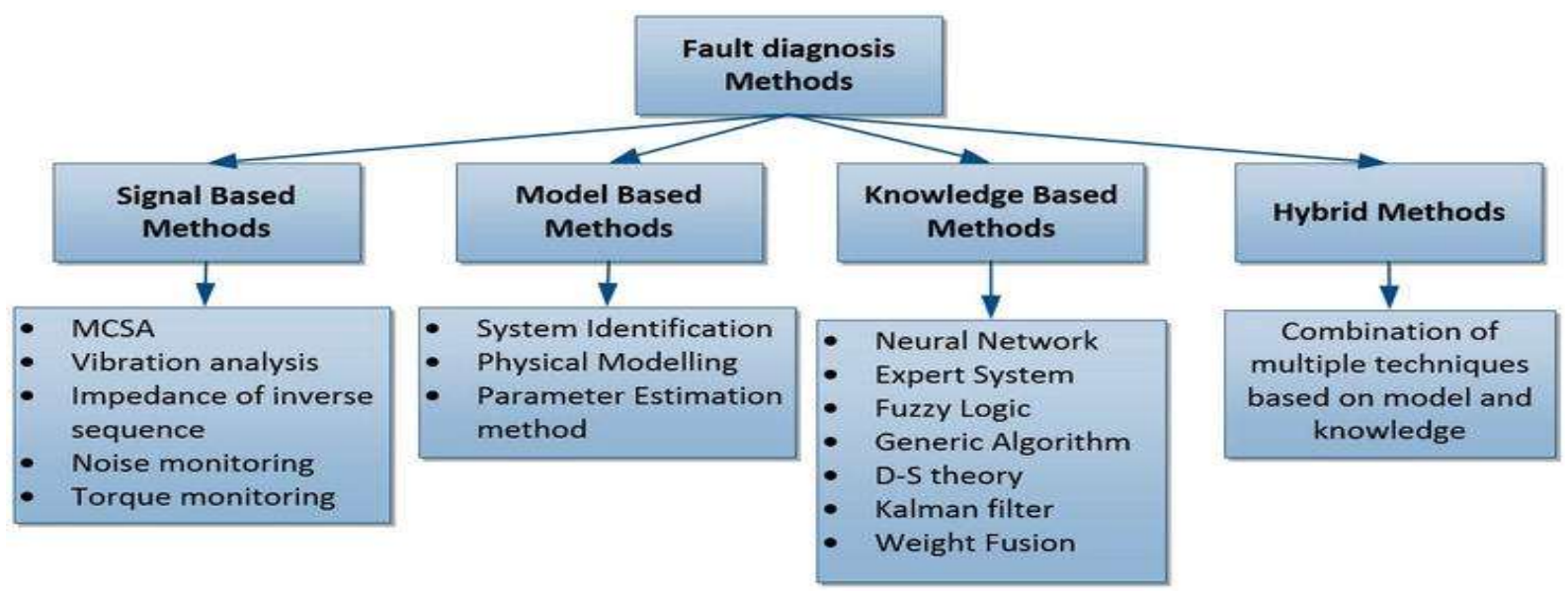

Figure 2.1: Various Fault detection and diagnostic methods.

Vibration analysis of gearbox is performed for two main reasons, first reason is to separate the gearbox related vibration signals from other components and reduce the noise that may be responsible for masking gearbox vibration signal particularly in the starting stage of fault. While the second reason is to know the status of the gearbox, it means that to differentiate between healthy and faulty gears. Some of the widely used techniques to accomplish these purposes are Waveform analysis, Faster Fourier Transform (FFT), Order analysis, probability density moments, Time-Frequency analysis, Spectral Analysis, and time-synchronous average. Because of the ease of measurement these vibration measurement techniques are most popular among all. Most modern gear diagnostic techniques are based on the examination of vibration signals acquired from gearbox casing. The gear meshing frequency (GMF) and even its harmonics, along with sidebands due to modulation events, are generally acknowledged to be the most essential components in gear vibration spectra [10]. The increases in the number, as well as the amplitude of these sidebands, might indicate a fault occurrence. Furthermore, the spacing between the sidebands is proportional to their source.

\section{DETECTION AND DIAGNOSTIC OF GEARBOX FAILURE WITH VARIOUS VIBRATION ANALYSIS TECHNIQUES.}

The primary reasons for gear failure are as follows: (i) design flaw;(ii) manufacturing error; and (iii) application error. Design flaws can occur as a result of factors such as inadequate gear geometry, the use of inferior materials, quality, maintenance, and other criteria. Manufacturing errors may have been in the form of machining faults or heat treatment issues. Application errors can occur as a result of problems such as vibration, installation and assembly, cooling, and maintenance. Table 2 provides an overview of the modes of safetycritical failure. Several scholars researched using vibration analysis to detect and diagnose gearbox defects. Several signal processing techniques such as time domain, time-frequency domain, frequency domain, wavelet transform are implemented and tested. 
Table 2: Modes of safety critical failure

\begin{tabular}{|c|c|c|c|}
\hline Failure & Failure mode & Cause & Contributing factors \\
\hline \multirow{5}{*}{$\begin{array}{l}\text { Shaft } \\
\text { fracture }\end{array}$} & \multirow{3}{*}{ Fatigue } & Unbalance & \\
\hline & & Misalignment & Coupling, bearing failure \\
\hline & & Bent shaft & \\
\hline & \multirow{2}{*}{ Overload } & Interference & Incorrect assembly, bearing failure \\
\hline & & Operational & \\
\hline \multirow{3}{*}{$\begin{array}{l}\text { Gear } \\
\text { fracture }\end{array}$} & \multirow{2}{*}{ Fatigue } & Life limit exceeded & \\
\hline & & Surface damage & \\
\hline & Resonance & Design & \\
\hline \multirow{6}{*}{$\begin{array}{l}\text { Tooth } \\
\text { fracture }\end{array}$} & \multirow{3}{*}{ Bending fatigue } & Life limit exceeded & \\
\hline & & Surface damage & Process related \\
\hline & & Thin tooth & Excessive wear, destructive scoring \\
\hline & Random fracture & Surface damage & Process related, foreign object, pitting/spalling \\
\hline & \multirow{2}{*}{ Overload } & Interference & Incorrect assembly, bearing failure \\
\hline & & Operational & \\
\hline \multirow{3}{*}{$\begin{array}{l}\text { Over- } \\
\text { heating }\end{array}$} & \multirow{2}{*}{ Lubrication } & Insufficient oil & \\
\hline & & Loss of oil & Oil line failure, filter bowl failure \\
\hline & Insufficient cooling & Cooling fan failure & Shaft/gear fracture \\
\hline
\end{tabular}

\subsection{Time Domain Analysis.}

To identify a defect in a gear-rotor-bearing system, time domain techniques analyse both amplitude and phase characteristics of the vibration time signal. The time domain is indeed a natural perception that offers physical insight into vibration [11]. It is especially effective in assessing impulsive information from bearing and gear faults that have non-steady and brief transient impulses [12]. If properly understood, time-domain vibration signals may produce a huge quantity of information. This method can highlight some features that are not readily apparent. In the monitoring of machine conditions, several time-domain approaches can be utilised and some of these are as follow.

\subsubsection{Waveform analysis}

Time waveform data analysis is not really a new approach. Time waveform data was viewed on oscilloscopes in the earlier years of vibration analysis, and frequency components were computed by hand. Waveform analysis involves recording the event's time history on either a storage oscilloscope or even a real-time analyser. Aside from evident fundamental signal appreciation, it is valuable in the analysis of non-steady states and small transient impulses. Waveform analysis may also be used to determine non-synchronous vibrations along with shaft speed, and in machinery coast down the investigation, the waveform can reveal the existence of resonance. In the following scenarios, the time waveform may be utilised to effectively enhance spectral information; (i) Applications requiring low speeds (less than 100 RPM); (ii) Gears (iii) Looseness (iv) Rubs (v) Beats (vi) X-Y probed sleeve bearing machines. 
The existence of resonance can be indicated by the waveform using machine cost down an assessment. The digitised data of such 8202 samples is employed to transfer in the time domain signature using MATLAB algorithms with a time length of 1.934 seconds. The spectrum of faulty gear system of vibration signals can be seen in Figure 3.1.1.

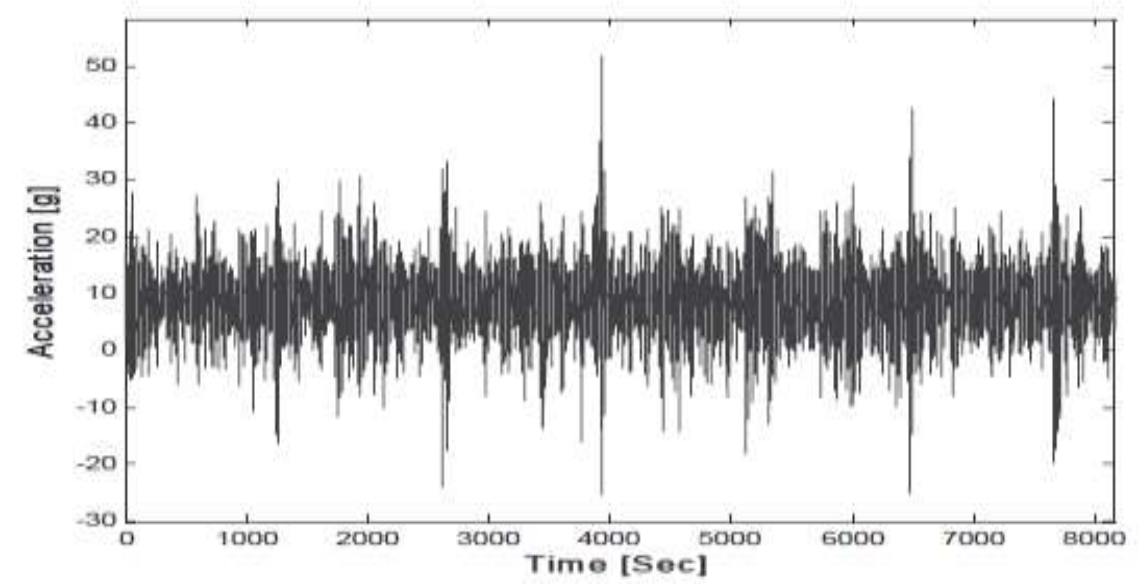

Figure 3.1.1:Vibration waveform for a defective gearbox with a period of up to 1.934 seconds.

\subsubsection{Indices}

In vibration analysis, indices are indeed employed [13,14]. To measure the time signal, peak value, average value, standard deviations, root-mean-square (RMS) value, kurtosis and also their ratio crest factor are frequently employed. Because peak value is not related to statistical quantity, this might not be credible in identifying damage of consistently operating systems. But RMS value, on the other hand, is better suitable for steady-state systems. The term crest factor is described as the measure of peak to RMS value, has been recommended as a popular parameter since it contains both characteristics that are used in vibration analysis. Crest factors are only reliable when there is significant impulsiveness [15]. Crest factors of gear in excellent condition typically range from 3.7 to 4.0, whereas values for gear having impulsive nature of faults can reach 10.0. Crest factors greater than 4.5 are often indications of deterioration [16]. The vibration signal crest factor values are not sensitive enough towards the working load and speed of gear unless the speed required to produce a gear vibration exceeding ground noise level is sustained, and an adequate load is applied that maintains complete contact. The peak well as RMS values rise proportionately as the working speed increases, resulting in a consistent crest factor. The reliability of the technique of crest factors to identify gear defects collapses mostly in absence of substantial impulsiveness.

$$
\text { Peak Value }=\mathrm{A}_{\max }
$$

$$
\mathrm{RMS}=\sqrt{\sum_{\mathrm{n}=1}^{\mathrm{N}} \frac{[\mathrm{A}(\mathrm{n})]^{2}}{\mathrm{~N}}}
$$


Here Amax = highest amplitude value within time domain; $A(n)=$ within time domain, the amplitude of nth digitised point; $\mathrm{N}=$ represents the number of time-domain points. The RMS technique is the easiest way to measure faults in the time domain. However, mostly in the early phases of gear and bearing deterioration, the RMS level may not indicate any significant variations. A better indicator is the "crest factor," which is defined as the ratio of the input signal's peak level to its RMS value [17]. As a result, when the time series signal reaches a peak, the crest factor value rises. This feature is utilised to detect variations as in signal patterns caused by impulsive vibration sources like gear tooth breakage or indeed a fault on a bearing's outer race. The crest factor strategy is not considered to be especially sensitive. The crest factor's equation is as follows:

Crest factor=peak level/RMS level.

\subsubsection{Statistical methods}

Statistical analyses on time-domain data may also be performed. The probability density represents the probability of detecting instantaneous values inside a particular amplitude interval, divided by interval size. All signals would have their own characteristic probability density curve. These curves can be utilised in equipment condition monitoring if they are obtained from the machinery vibration signals.

\subsubsection{Probability density moments}

A sequence of single-number indices can indeed describe the shape of the probability density curve. These are just the moments of the curve which are analogous to mechanical moments of the plane's centroid.

\subsubsection{Kurtosis (K)}

Kurtosis is the signal's normalised fourth statistical moment and assesses the relative flatness or peakedness of a distribution in relation to the typical normal distribution [18]. Kurtosis measures the size of tail and used as an indicator of the tallest peak in each set of data. The kurtosis equation is given as:

$$
K=\sum_{n=1}^{N} \frac{[y(n)-\mu]^{4}}{N \times \sigma^{4}}
$$

Where $y(n)=$ data $(n=1,2,3, \ldots, N) ; N=$ Total data sample number; $\mu=$ mean; while $\sigma=$ standard deviation.

\subsubsection{Skewness (S)}

Skewness is an asymmetry measure, or perhaps, lack of symmetry. The data set distribution is symmetric if it appears like the left and right sides of the central point. The skewness is calculated using Equation (5). 


$$
S=\sum_{n=1}^{N} \frac{[y(n)-u]^{3}}{N \times \sigma^{3}}
$$

\subsection{Frequency domain analysis}

Fast Fourier transform (FFT), Power Cepstrum Analysis, Hilbert Transform Method are some of frequency-domain techniques found very useful in rotating machines condition monitoring particularly gearbox fault analysis. These techniques use the variation in power spectral density of signal caused by a faulty gear and/or bearing to determine element damage [19]. Any legitimate signal may be broken down into a series of distinct sine waves. Each sine wave extracted from the signal shows in the frequency domain as vertical lines. Its amplitude is represented by its height, and its frequency is represented by its location. The signal is the frequency domain representation of a signal. The vibration has been entirely defined by the frequency domain. The frequency-domain analysis helps not only to finds rotating machinery faults, also reveals the cause of defects in rotating machinery [20].

\subsubsection{Fast Fourier transform (FFT)}

Fast Fourier Transform (FFT) includes essentially a set of specific algorithms which execute the discrete Fourier transformation with a significant reduction in computational time. It is important to note that the FFT is not a separate transform from the DFT, but only a means of determining the DFT with a significant decrease in the number of computations necessary. The discrete Fourier transform (DFT) will be used to analyse a signal which exists in discrete-time form. The equation for DFT is as follows:

$$
X(m)=\frac{1}{N} \sum_{n=0}^{N-1} x(n) e^{-J 2 \Pi m n / N}
$$

Here $\mathrm{m}=0,1,2, \ldots, \mathrm{N}-1$. Also, a total number of samples in a particular time record is denoted by $\mathrm{N}$. Where $\mathrm{x}(\mathrm{n})$ represents discrete-time function and $\mathrm{X}(\mathrm{m})$ is represents the DFT of the signal(function). The DFT method lies at the heart of digital signal analysis, discrete Fourier transformation DFT method is the foundation of digital signal investigation, and it requires complicated multiplications to create a specific N-point transform. computations are necessary if averaging across $M$ time signals is desired. The Fast Fourier Transform (FFT) algorithm decreases the number of calculations needed significantly. It's simply a more efficient DFT evaluation process. The discrete Fourier transform DFT transform may be used to transform any complicated valued series, it might take an unexpected long time to analyse a large series, and time proportionate to the square of, the total numbers of data points lies in the series. Cooley \& Tukey developed the much quicker FFT algorithm (1965). The most popular version of this method (Radix-2 Cooley-Tukey) has only one stipulation: that amount of points within the series must be a power of two. The time it takes to compute the FFT is equivalent to $\mathrm{N} \log 2(\mathrm{~N})$. Thus, a transform upon 1024 data points using the discrete Fourier transform (DFT) took approximately 100 times longer than using the FFT, indicating a 
significant performance gain. The rotating machine vibration characteristics are unique to some extent, and this is due to the varied transfer characteristics of these rotating machines.

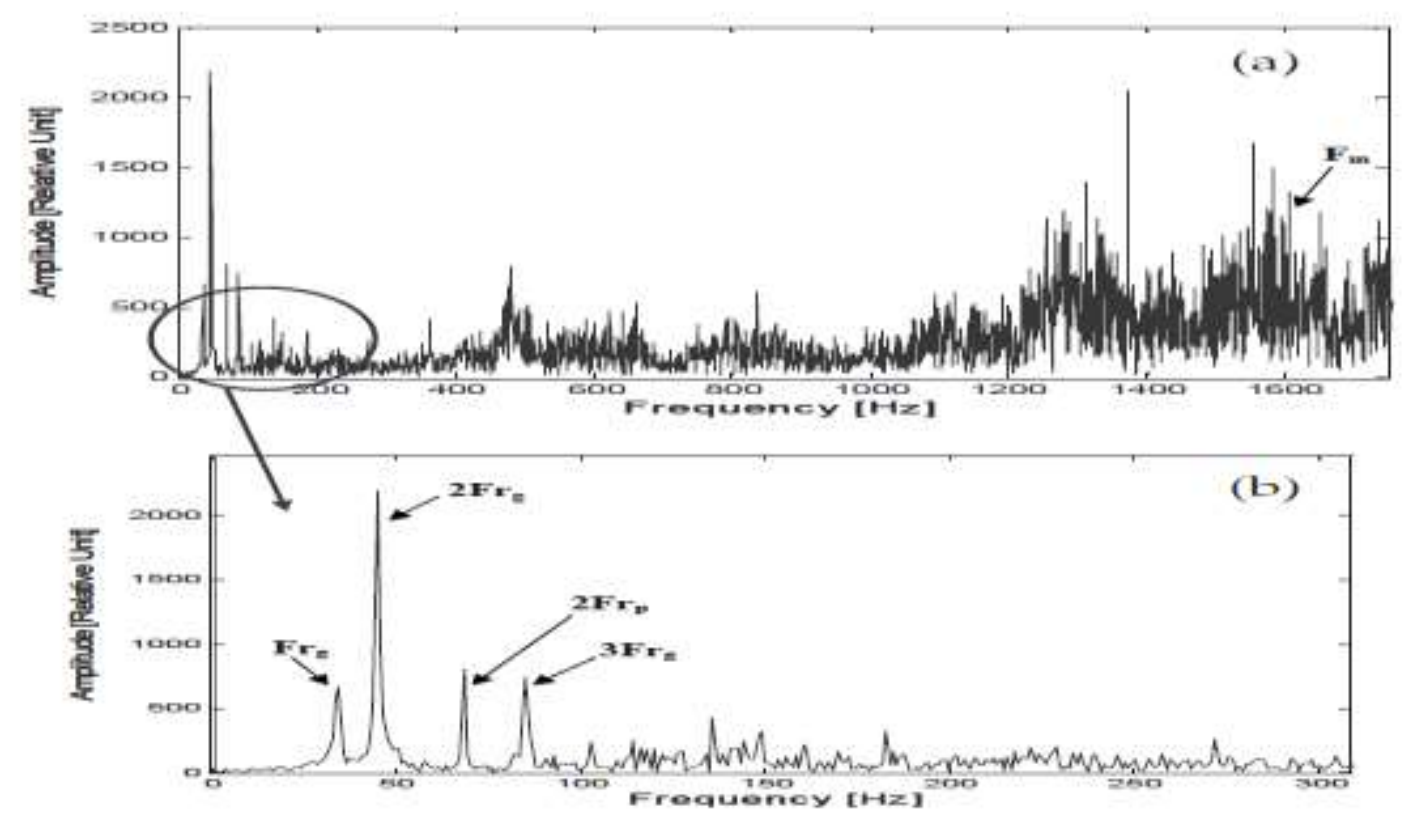

Figure 2.2: (a) Defective gear vibration signal's FFT spectrum.(b) Zoom view of gear vibration signal's FFT spectrum.

The FFT plot shows different big and tiny peaks corresponding to distinctive frequencies that reveal the origin of faults; alternatively, the FFT plot displays frequencies in relation to shaft harmonics. Special consideration must be paid to the bearing fault frequencies in the FFT spectrum when dealing with gear faults. Peaks in the FFT spectra would occur at recognised fault frequencies. These spikes(peaks) may or may not reflect the identified fault. To determine whether the identified frequencies are produced by the indicated fault, one must go through their harmonics:

- When a peak occurs at fundamental defect frequency and then another peaking occurs at double the fundamental fault frequency, this is a strong sign suggesting perhaps the fault is real.

- If no peak emerges at the fundamental defect frequency, yet peaks arise at two, three, or possibly four times the fundamental frequency, it is also a strong indicator that the detected fault is real.

In figure 2.2(a) shows the frequency spectrum of a defective gear vibration signal. The peaks in this figure are at $F_{m}$ as well as its, second multiple frequencies. However, there are a few additional peaks of signals, because to the modulation effect. The peaks in Figure 2.2(b) are detected at $\mathrm{Fr}_{g}$ and $\mathrm{Fr}_{P}$, including its second as well as third multiple frequencies. However, there are also some additional peaks of signals because of the effect of modulation frequencies. These peaks are a strong indicator that the gear is defective, with the nature of the defect being chipped(broken) tooth pitch to top at $2 \%$ of thickness. FFT for determining the intensity of the fault: 
- One method for determining the intensity of a fault is to evaluates its amplitude to previous readings collected under identical conditions.

- Another method is to evaluate the amplitude to other measurements acquired from similar machines operating under identical conditions. A reading that is greater than usual suggests a problem.

\subsubsection{Frequency band Analysis}

Another method for limiting the amount of data accessible in a spectrum to acceptable proportions is passband analysis. This technique entails the monitoring of a broad or narrow spectrum of frequencies, wherein component defect frequencies are expected. Discrete frequency monitoring is the term for this technique. The frequencies in which bearing defects are detected are indeed the modulation frequency, as the resonance frequency of housing is higher and there are limited spectral lines. The sidebands are difficult to resolve if it is not impossible. The passband approach allows for direct measurement of the modulating signal's frequency. The objective of bandpass filtration is to eliminate or reject signals of low frequency and high amplitude associated with misalignment, unbalance, and looseness. The transfer function may be defined to provide a better design. In the frequency domain, filtering is a process, where the components of frequency are further analysed and are multiplied by one and the remaining components of frequency are then multiplied by zero. Equation (7) may be used to define the filter function mathematically:

$$
\begin{aligned}
& H(\omega)=1, \omega_{1}<\omega<\omega_{2} \\
& H(\omega)=0, \text { otherwise }
\end{aligned}
$$

Thus, a bandpass filtration approach is used to obtain the features of the defective gearbox for further defect categorization. There are several kinds of filters available, namely Butterworth, Bessel, Elliptic and Chebyshev. Elliptic filters have sharper roll-off characteristics as compared to Butterworth or Chebyshev filters, but they are evenly rippled in the stop and passbands. Elliptic filters, in general, have any filter type of lowest order and fulfil stated performance specifications.

\subsubsection{Spectral Analysis}

Spectral analysis is typically inefficient in detecting early-stage gear faults. As a result, several researchers have suggested the use of alternative vibration analysis techniques should be used to detect defect indications as earlier. The most widely used vibration analysis approach for predictive maintenance in geared power transmission is spectral analysis, which has been shown to be a useful tool for detecting and diagnosing defects in basic rotating machinery [21]. The essential process common in all spectrum analysis strategies is the transformation of a vibration signal's time-domain representation into a frequency - domain. It can be accomplished via the application of narrowband filtration or, more recently, using the DFT of digital data. The level of vibration at each frequency reflects the vibration throughout a narrow frequency range centred on the specified frequency with a bandwidth set by the conversion technique employed. For machines working at a given constant speed, the vibrations frequencies generated by the several moving parts can be estimated; hence, a shift 
in the level of vibration within a specific frequency band can frequently be connected with a specific machine component. Analysis of relative levels of vibration in different bands of frequencies can usually provide an insight into the type of defect, offering some diagnostic capabilities. The vibration signal frequency domain spectrum reveals the frequency characteristic of vibrations, if impulse occurring frequencies are near to being, one of gear distinctive frequencies such as pinion frequency, gear frequency, and gear mesh frequency as shown in the below equations $(8,9,10)$. Then it could be an indication of a gearbox fault the frequency $\left(F r_{g}\right)$ of gear is computed as follows:

$$
F r_{g}=R_{g} / 60[H z]
$$

The frequency of pinions $\left(F r_{P}\right)$ is calculated as follows:

$$
F r_{P}=R_{P} / 60[\mathrm{~Hz}]
$$

The frequency of tooth mesh $\left(F r_{m}\right)$ is calculated as follows:

$$
F r_{m}=F r_{P} N_{P} \quad[H z]
$$

here $R_{g}$ is indeed the gear speed (rpm), number of pinion teeth are denoted by $N_{P}$, pinion speed in rpm is $R_{P}$, and number of gear teeth denoted by $N_{g}$.

\subsection{Order Analysis}

Vibration and noise signals in reciprocating or rotating machinery are analysed utilizing order analysis. Some of notable example of reciprocating or rotating machinery includes compressors, automobile engines, pumps, aircraft, and turbines. Such machinery generally contains variety of mechanical components such as bearing, shaft, gearbox, coupling, blades, and belt. When machinery operates, each and almost every mechanical component generates its own sets of unique vibration and noise patterns. Each mechanical part offers a distinct component to the total machine vibration and noise. This technique is beneficial for analysing vibration and noise signals, whenever the rotational speed fluctuates. Order is stated as the rotational speed normalization. The first order should be rotational speed and then, order $n$ is the $n$-times of the rotational speed. The vibrational harmonics of rotational speed are known as order components. Order analysis can be able to provide information about specific mechanical components and also about the whole machinery. Order analysis includes a form of analysis devoted to the study of rotating machinery and how their frequencies are altered as the machine's rotating speed varies. It resamples the raw signals from the time domain into the angular domain, aligning the given signal with the angular position of the machine. This eliminates the effect of shifting frequencies here on the FFT method, which is usually incapable of handling such situations. Mechanical components including shafts, gearboxes, coupling, and bearing are used in a revolving or reciprocating machinery. The overall conditions of machines are mostly governed by the quality of the mechanical parts. The machinery will generate very minimal vibrations if the mechanical components are adequately tuned for vibrations. On the other hand, mechanical faults will usually cause the equipment to vibrate substantially. As a result, machinery vibrations can be utilised as a reliable indicator of mechanical faults. Some of the most commonly found mechanical faults 
are misalignment, mechanical looseness, resonance, imbalance, gearbox defects, and bearing faults exhibit unique vibration characteristics. The interconnections between conventional mechanical defects and vibration components are summarised in Table 3.

Table 3: Vibrational characteristics of mechanical defects

\begin{tabular}{|c|l|l|}
\hline S. No. & Mechanical faults & Vibration components \\
\hline 1 & Imbalance & $1 x$ component \\
\hline 2 & Misalignment & $1 x$ and $2 x$ components. \\
\hline 3 & Mechanical looseness & Harmonics of $1 x$ and $0.5 x$ components \\
\hline 4 & Resonance & $\begin{array}{l}\text { High vibration amplitude and large phase change at certain } \\
\text { speed range }\end{array}$ \\
\hline 5 & Gear defect & $\begin{array}{l}\text { Gear mesh } n x \text { components ( } n \text { is the number of gear teeth), } \\
\text { usually modulated by rotational speed components }\end{array}$ \\
\hline 6 & Rolling-element bearing defect & $\begin{array}{l}\text { Non-synchronous vibration components, usually } \\
\text { modulated by rotational speed components }\end{array}$ \\
\hline \multicolumn{2}{|l|}{ Note: $1 x$ means first-order component and $n x$ means $t^{\text {th }}$ order component. } \\
\hline
\end{tabular}

The most essential information regarding the machine is generally the noise as well as vibration components of any potential mechanical faults. In most the applications, the primary objectives in noise and vibration analysis are to extract patterns from vibration and noise signals and then use these patterns to examine the condition of mechanical parts. Order analysis can assist you in obtaining those distinguishing characteristics. A typical order analysis application involves the following steps:

1. Obtain vibration or noise signals, as well as a tachometer signal.

2. Pre-Processing of the vibration or noise signals.

3. Process the signal of the tachometer to obtain the profile of rotational speed.

4. Execute the vibration or noise signals order analysis and also speed profiling.

5. Display outcomes of analysis in various formats. 


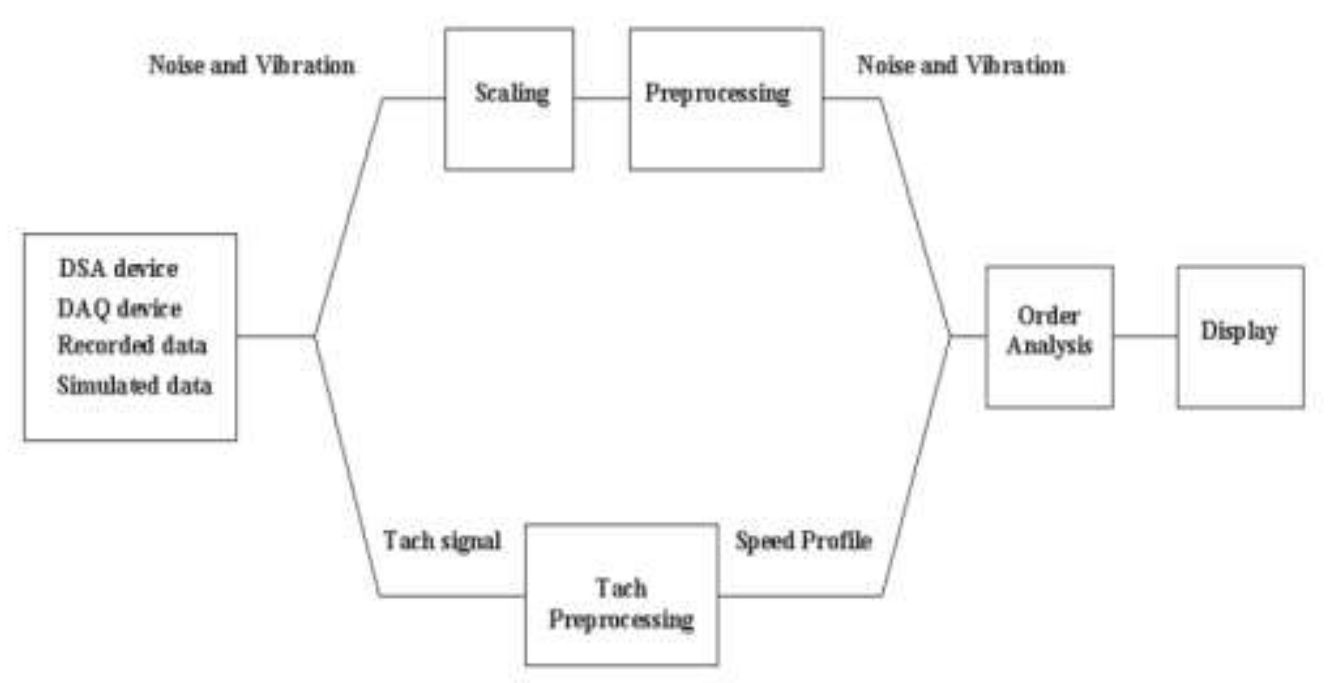

Figure 3.3: Deployment procedure for common order analysis.

\subsection{Time synchronous averaging}

Stewart (1977) demonstrated that by TSA it is possible to reduce a complicated time-domain vibration signal taken from the transmission, and then reduced it to forecasts the vibration for particular shafts including their associated gear. TSA is a substantially different method than the typical spectrum averaging employed in FFT analysis. Since the averaging is done only in the time domain rather than the frequency domain, TSA offers the time domain signal with exceptionally low noise. The main concept behind the synchronous signal averaging would be that all vibration associated with a shaft, and also with the gears on such shaft will repeat on a regular basis with the rotation of the shaft. The vibration, whose nature is periodic with the rotation of shaft will be reinforced, while the vibrations that might not be periodic with the rotation of shaft will seem to terminate out, thus by segregating the vibration data into contiguous sections, each being exactly one shaft period in length, and also ensembleaveraged nearly a reasonably big number of segments. Thus, leaving a signal that representing the average vibration during one shaft revolution. Figure 4 shows that how this approach might be implemented on a steady time signal from the gearbox, with each rotational cycle of the shaft calculated by the use of a tacho multiplier. The process can be carried out either in the frequency or time domain (as far as averaging of the frequency domain is conducted out on the complex frequency domain representation). It should be noted that the approach can be carried out in either the frequency or time domain (as far as frequency domain average is carried out on the sophisticated frequency domain representation). The term time-domain averaging (Braun, 1975; McFadden, 1986b ) was adopted to separate the approach from power spectra or averaging of amplitude to reduce the variance in spectral assessment (Randall, 1987). TSA also becomes useful in the case of waveform analysis of machinery vibration, particularly with the gear drives. The trigger in this situation is obtained by a tachometer that delivers one pulse per gear revolution. Stewart (1977) devised a set of dimensionless parameters based upon synchronous signal averaging, which he labelled Figures of Merit (McFadden, 1986b). Commonly described as a hierarchical class, Stewart presented a methodology using above mentioned dimensionless parameters (Figure of Merits) for the identification as well as partial faults diagnostic 


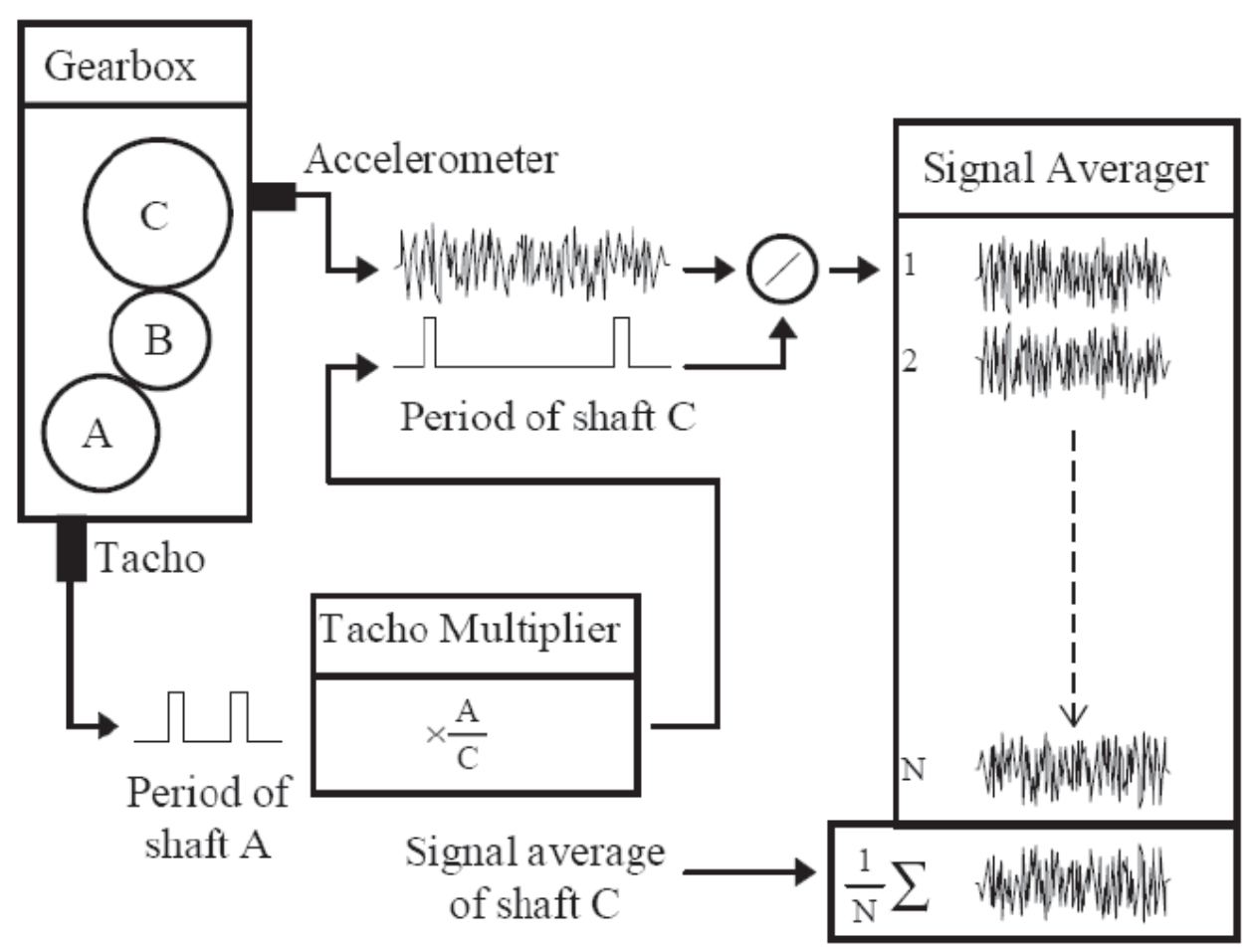

Figure 4: Synchronous signals averaging.

\subsubsection{FM0}

Stewart (1977) introduced "FM0" parameter as a reliable indicator of gear mesh faults. FM0 is a straightforward way of detecting major variations in the meshing patterns. Major tooth faults often increase overall level of peak-to-peak(ptp) signal but have no effect on the meshing frequency. FM0 is described as the TSA signal's peak-to-peak threshold divided by the sum of the intensity(amplitude) at gear mesh frequency and its harmonics. Peak-to-peak stays unchanged during heavy wear, whereas meshing frequency drops, enabling the FM0 factor to climb. Unfortunately, FM0 is not really a reliable predictor for slight tooth damage. FM0 formula is denoted as:

$$
F M 0=\frac{P P A}{\sum_{i=1}^{n} A\left(F_{i}\right)}
$$

Here PPA denotes peak to peak(ptp) amplitude of time synchronized average waveform. $\mathrm{A}\left(F_{i}\right)$ is just amplitude of gear meshed fundamental including harmonics.

\subsubsection{FM4}

Stewart (1977) developed FM4 parameter to enhance FM0 by identifying faults localized to limited teeth. This is achieved by first creating the difference signal(d) as shown in equation (15). After that normalised kurtosis of ' $d$ ' is calculated.FM4 is represented in below equation. 


$$
F M 4=\frac{N \sum_{i-1}^{N}\left(d_{i}-\bar{d}\right)^{4}}{\left[\sum_{i=1}^{N}\left(d_{i}-\bar{d}\right)^{2}\right]^{2}}
$$

Here $\bar{d}$ is the average of difference signal, while $\mathrm{N}$ denotes the maximum count of data points.

\subsubsection{NA4}

Zakrajsek (1994) developed a parameter named "NA4". Which is a generic fault indicator, that responds to not only the onset of deterioration, but also with the fault's continued growth. NA4 is computed by dividing fourth statistically moment of the residue signal by the latest run time average variance of residual signal, increased to second power. The expression for NA4 is given below.

$$
N A 4=\frac{N \sum_{i=1}^{N}\left(r_{i M}-\overline{r_{M}}\right)^{4}}{\left\{\frac{1}{M} \sum_{j=1}^{M}\left[\sum_{i=1}^{N}\left(r_{i j}-\bar{r}_{J}\right)^{2}\right]\right\}^{2}}
$$

Here $r$ represents the mean of residual signal, the maximum number of points of data in time signal is denoted by $\mathrm{N}, \mathrm{M}$ is the current time signal's number, where $\mathrm{j}$ represents index of run ensemble's time signal. NA4 is a non-dimensional parameter, similar to FM4, and is designed to possess a conventional value of 3 , provided $r$ is Gaussian in nature.

\subsubsection{M6А}

Martin (1989) proposed the use of M6A factor, which is act as a tool to indicate the surface defects exists on the machine components. Due to the usage of sixth moment, it is envisaged that M6A are more responsive to spikes in the disparity signal. M6A is written as follows:

$$
M 6 A=\frac{N^{2} \sum_{i=1}^{N}\left(d_{i}-\bar{d}\right)^{6}}{\left[\sum_{i=1}^{N}\left(d_{i}-\bar{d}\right)^{2}\right]^{3}}
$$

The moment get normalised by the cubed of the variance in this instance.

\subsubsection{M8A}

Martin also proposed the M8A parameter (1989), is claimed to be somewhat more reactive to peaks throughout in case of difference signals than that of M6A. M8A employs the eight moments to get normalised up to fourth power by the variance and is written as follows:

$$
\mathrm{M} 8 \mathrm{~A}=\frac{N^{3} \sum_{i=1}^{N}\left(d_{i}-\bar{d}\right)^{8}}{\left[\sum_{i=1}^{N}\left(d_{i}-\bar{d}\right)^{2}\right]^{4}}
$$

\subsubsection{NA4*}

Decker et al (1994) developed again the parameter NA4* as an improvement to NA4. The denominator part of NA4 parameter is statistically changed in NA4*. Once the variance of remnant signal crosses a predetermined statistical value, then averaging ends and also the 
denominator term get fixed. This adjustment was driven by the fact that when deterioration moves from confined to dispersed region, there is subsequent increase in the signal's variance, causing the kurtosis to restore to standard values after the early indication of damage.

$$
N A 4^{*}=\frac{\frac{1}{N} \sum_{i=1}^{N}\left(r_{i}-\bar{r}\right)^{4}}{\left(\widetilde{M}_{2}\right)^{2}}
$$

where $r$ denotes the signal's residual, also the mean of residual signal is denoted by $r$, and $\mathrm{N}$ represents maximum number of points of data in a particular time record, $\widetilde{M}$ denotes the residual signal's variance.

\subsubsection{Demodulation}

Demodulation techniques are very useful for identifying and diagnosing amplitude modulation factors caused by gear deterioration in the vicinity of a singular gear mesh frequency (1GMF) or, for some situations, a dual gear mesh frequency (2GMF). Stewart's (1977) insight that gear tooth breakage increased the amplitude of sidebands around the typical meshing components prompted subsequent research into the nature of amplitude, and also phase modulation algorithms. Demodulations differ significantly from enveloping strategies because in demodulation strategies the cumulative effect over the frequencies range can be separated. The original data(raw) can be demodulated by the use of high pass filtrations about at 85 per cent of gear meshed frequency. After that raw data is low pass filtered at about 115 per cent of gear meshed frequency. Here power spectral density belongs to filtered data(signal) may be used to determine the real gear mesh frequency.

\section{CONCLUSIONS}

- This paper provides a brief overview of some of the existing vibration-based methods being used for geared condition monitoring. After going through a brief review of the literature about geared fault analysis, the relevant conclusions are gained as:

- Vibration signals from gearboxes are typically periodic and chaotic. The timefrequency domain averaging technique substantially suppresses noise from the data(signal).

- Use of spectral analysis in the identification and diagnostic of shaft faults may be worthwhile.

- FFT has been able to demonstrate the impulses at the defect characteristics frequencies and its numerous frequencies within the frequency domain, but due to the effect of modulation, numerous different kinds of peaks are also present. Thus, it makes it hard to identify fault classifications using this technique.

- When using bandpass analysis to analyse gear vibration signals, it is determined that this technique is helpful for extracting features for the fault diagnosis. The root means square(RMS) value of the filtered signal across three frequency bands was found to be a significant feature for developing intelligent systems using ANN. 
- The synchronous signal averaging does have the potential to dramatically simplify the detection of gear and shaft faults (critical safety failures) by attenuating nonsynchronous oscillations and signals to the point where optimal filtering can be applied. Further, more development required to be done for the deployment of synchronous averaging algorithms, as well as analysis of their results.

- By the use of feature extraction from the vibration signal, a specialist system relying on fuzzy logic and ANN could be formed for the comprehensive fault classification.

All such conclusions could be helpful to encourage more investigation and more parameters needed to develop that contained robust expert system and should be helpful to diagnose various problems with gearbox more precisely and efficiently. It has been demonstrated that by using these methods of vibration signal assessment, signal flaws and dispersed flaws in gearboxes can be detected. A tooth crack, breakage, or spall in an internal or external race of a bearing are all examples of signal faults, while distributed defects are because of uneven wear (scuffing, pitting abrasion, erosion).

\section{REFERENCES}

[1] Zakrajsek, J.J.: “An Investigation of Gear Mesh Failure Prediction Techniques," NASA TM-102340, AVSCOM TM89-C-005, November 1989.

[2] Lebold, M.; McClintic, K.; Campbell, R.; Byington, C.; Maynard, K.: "Review of Vibration Analysis Methods for Gearbox Diagnostics and Prognostics," Proceeding of the 54th Meeting of the Society for Machinery Failure Prevention Technology, Virginia Beach, VA, pp. 623-634, May 1-4, 2000.

[3] Swansson, N.S.: “Application of Vibration Signal Analysis Techniques to Signal Monitoring," in: Conference on Friction and Wear in Engineering, Institute of Engineering, Barton, Australia, pp. 262-267, 1980.

[4] Zakrajsek, J.J.: "A Review of transmission Research at NASA Lewis Research Center," Technical Report NASA TM-106746, ARL-TR-599, NASA and the US Army Research Laboratory, December 1994.

[5] Brennan, M.; Chen, M.H.; and Reynolds, A.G.: "Use of Vibration Measurements to Detect Local Tooth Defects in Gears," Journal of Sound and Vibration, vol. 31, pp. 1217, November 1997.

[6] Forrester B.D.: “Time -Frequency Analysis in Machine Fault Detection,” Time Frequency Signal Analysis, B. Boashash Edition, Longman Cheshire, Melbourne, Australia, 1992.

[7] Newland, D.E.: “An Introduction to Random Vibration, Spectral, and Wavelet Analysis," 3rd Edition, Longman Essex, 1993.

[8] Miller,; Irwin,; and Freund, J.E.: "Probability and Statistics for Engineering," 3rd Edition, Prentice-Hall, Inc., New Jersey, p.74, 1985.

[9] Lebold, M.; McClintic, K.; Campbell, R.; Byington, C.; Maynard, K.: "Review of 
Vibration Analysis Methods for Gearbox Diagnostics and Prognostics," Proceeding of the 54th Meeting of the Society for Machinery Failure Prevention Technology, Virginia Beach, VA, pp. 623-634, May 1-4, 2000.

[10] Wang, Wilson Q.: "Rotary Machinery Health Condition Monitoring Fault Diagnosis and Prognosis," Thesis, University of Waterloo, Waterloo, Ontario, Canada, 2002.

[11] Stewart, R.T.: "Some Useful Analysis Techniques for Gearbox Diagnostics," Technical Report MHM/R/10/77,Machine Health Monitoring Group, Institute of Sound and Vibration Research, University of Southampton, July1977.

[12] Decker, H.J.; Handschuh, R.F.; and Zakrajsek, J.J.: “ An Enhancement to the NA4 Gear Vibration Diagnostic Parameter," Technical Report NASA TM-106553, ARL-TR-398, NASA and the US Army Research Laboratory,July 1994.

[13] McFadden, P.D.: "Detecting Fatigue Crack in Gears by Amplitude and Phase Demodulation of the Meshing Vibration," Journal of Vibration, Acoustic, Stress, and Reliability in Design, vol. 108, pp. 165-170, 1986.

[14] Wang, W.; and McFadden P.: "Early Detection of Gear failure by Vibration Analysis I, Calculation of the Time -Frequency Distribution," Mechanical System and Signal Processing, vol. 7, no. 3, pp. 193-203, 1993.

[15] Lin, J. \& Zuo, M. J. 2003, “Gear box fault diagnosis using adaptive wavelet filter”, Mechanical Systems and Signal Processing, Vol. 17, No. 6, pp. 1259-1269.

[16] Cooley, J. W. \& Tukey, J. W. 1965, “An Algorithm for the Machine Calculation of Complex Fourier Series”, Mathematics of Computing, Vol. 19, pp. 297-301.

[17] Randall, R. B. 1987, Frequency Analysis, 3rd edition, Brüel and Kjær.

[18] Michel, A. N. \& Miller, R. K. 1983, Gears and Their Vibration, Marcel Dekker, Inc.

[19] Randal, R. B. 1982, “A New Method of Modeling Gear Faults", Journal of Mechanical Design, Vol. 104,pp. 259-267.

[20] Michel, A. N. \& Miller, R. K. 1983, Gears and Their Vibration, Marcel Dekker, Inc.

[21] Stewart, R. M. 1977, "Some useful analysis techniques for gearbox diagnostics", Technical Report MHM/R/10/77, Machine Health Monitoring Group, Institute of Sound and Vibration Research, University of Southampton, July.

[22] A Aherwar (2012) An investigation on gearbox fault detection using vibration analysis techniques: A review, Australian Journal of Mechanical Engineering, 10:2, 169-183.

[23] Prabhakar, S., Mohanty, A. R., \& Sekhar, A. S. (2002). Application of discrete wavelet transform for detection of ball bearing race faults. Tribology International, 35,793-800.

[25] Vyas, N. S., \& Satish Kumar, D. (2001). Artificial neural network design for fault identification in a rotor bearing system. Mechanism and Machine Theory, 36,157-175. 
[26] Sawalhi N, Randall RB (2014) Gear parameter identification in a wind turbine gearbox using vibration signals. Mech Syst Signal Process 42:368-376.

[27] Polyshchuk VV, Choy FK, Braun MJ (2000) New gear-fault detection parameter by use of joint time-frequency distribution. J Propuls Power 16(2):340-346.

[28] Tandon N, Choudhury A (1999) A review of vibration and acoustic measurement methods for the detection of defects in rolling element bearings. Tribol Int 32(8):469480.

[29] Lei Y, Zuo MJ, He Z, Zi Y (2010) A multidimensional hybrid intelligent method for gear fault diagnosis. Expert Syst Appl 37(2):1419-1430.

[30] Wang W (2001) Early detection of gear tooth cracking using the resonance demodulation technique. Mech Syst Signal Process15(5):887-903.

[31] D. Goyal, B.S. Pabla, Condition based maintenance of machine tools - A review, CIRP Journal of Manufacturing Science and Technology, vol. 10, pp. 24-35, 2015.

[32] Vanraj, D. Goyal, A. Saini, S.S. Dhami and B.S. Pabla. 2016, April. Intelligent predictive maintenance of dynamic systems using condition monitoring and signal processing techniques - A review. In Advances in Computing, Communication, \& Automation (ICACCA) (Spring), International Conference on, pp. 1-6, IEEE, 2016.

[33] D. Goyal, B.S. Pabla, S.S. Dhami, K.C. Lachhwani, Optimization of condition-based maintenance using soft computing, Neural Computing and Applications, vol. 28(1), pp. 829-844, 2017.

[34] D. Goyal, Vanraj, B.S. Pabla, S.S. Dhami, Condition monitoring parameters for fault diagnosis of fixed axis gearbox: a review, Archives of Computational Methods in Engineering, vol. 23(4), pp. 543-556, 2016.

[35] S. Kumar, D. Goyal, R.K. Dang, S.S. Dhami, and B.S. Pabla. Condition based maintenance of bearings and gears for fault detection-A review. Materials Today: Proceedings, vol. 5(2), pp. 6128-6137, 2018.

[36] S. Kumar, D. Goyal, S.S. Dhami. Statistical and frequency analysis of acoustic signals for condition monitoring of ball bearing. Materials Today: Proceedings, vol. 5(2), pp. 5186-5194, 2018.

[37] R. Romary, R. Pusca, J.P. Lecointe et al., Electrical machine's fault diagnosis by stray flux analysis, Proc. Int. Conf. Electrical Machines Design Control and Diagnosis (Wemdcd), pp. 247-256, 2013.

[38] R.S. Marino, S. Peresada and P. Valigi, Adaptive Input-Output Linearizing Control of Induction Motor, IEEE Transaction on Automatic Control, vol. 38(2), pp. 208-221, 2014. 